\title{
Latest Studies of the SM Higgs Boson Couplings to Fermions at ATLAS
}

\author{
Yanlin Liu ${ }^{\mathrm{a}}$, on behalf of the ATLAS Collaboration
}

${ }^{a}$ University of Michigan

\begin{abstract}
This proceeding presents the latest studies on the Yukawa couplings of the Standard Model Higgs boson with 139 $\mathrm{fb}^{-1}$ data collected using the ATLAS detector at a center-of-mass energy of $13 \mathrm{TeV}$. A first direct probe of $C P$ violation in the top-quark Yukawa coupling using events where the Higgs boson is produced in association with top quarks $(t \bar{t} H$ and $t H)$, and decays into two photons $(H \rightarrow \gamma \gamma)$ is discussed. The latest results on the Higgs boson production in association with a $W$ or $Z$ boson $(V H)$ in the $H \rightarrow b \bar{b}$ channel are depicted as well. Finally, the searches for Higgs boson decays into two muons $\left(H \rightarrow \mu^{+} \mu^{-}\right)$and two electrons $\left(H \rightarrow e^{+} e^{-}\right)$are presented.
\end{abstract}

Keywords: Higgs boson, fermion, Yukawa coupling

\section{Introduction}

Since the discovery of the Higgs boson in 2012 [1, 2], study on its couplings to the fermions (Yukawa couplings), becomes one important sector at the Large Hadron Collider (LHC) experiments. According to the Standard Model (SM), the Yukawa coupling strength is proportional to the mass of the corresponding fermion. Any experimental deviation would be a sign of new physics beyond the SM. From 2015 to $2018,139 \mathrm{fb}^{-1}$ of $\sqrt{s}=13 \mathrm{TeV}$ proton-proton collision data was recorded by the ATLAS detector [3]. Based on this amount of dataset, studies on $t \bar{t} H / t H$ via $H \rightarrow \gamma \gamma[4]$ and $H \rightarrow b \bar{b}$ in the $V H$ production mode [5, 6] (third generation), as well as $H \rightarrow \mu^{+} \mu^{-}$[7] (second generation) and $H \rightarrow e^{+} e^{-}$[8] (first generation) are performed. Their latest results are reported in the following sections.

\section{2. $t \bar{t} H / t H$ via $H \rightarrow \gamma \gamma$ Channel}

The Higgs boson production in association with top quarks provides a direct access to the top-quark Yukawa

\footnotetext{
*Talk given at 23rd International Conference in Quantum Chromodynamics (QCD 20), 27 October - 30 October 2020, Montpellier France

${ }^{* *}$ Copyright 2020 CERN for the benefit of the ATLAS Collaboration. CC-BY-4.0 license.
}

coupling, as well as an opportunity to probe the charge conjugation and parity $(C P)$ properties of the Higgs boson interactions with the top quarks. The effective field theory used for this study is provided by the Higgs Characterization model [9]. Within this model, the term in the effective Lagrangian that describes the top-quark Yukawa coupling is:

$$
\mathcal{L}=-\frac{m_{t}}{v}\left\{\bar{\psi}_{t} \kappa_{t}\left[\cos (\alpha)+\mathrm{i} \sin (\alpha) \gamma_{5}\right] \psi_{t}\right\} H
$$

where $m_{t}$ is the top quark mass, $v$ is the Higgs vacuum expectation value, $\kappa_{t}(>0)$ is the top-quark Yukawa coupling parameter, and $\alpha$ is the $C P$-mixing angle. The SM corresponds to a $C P$-even coupling with $\alpha=0$ and $\kappa_{t}=1$.

Events are selected by requiring two isolated photon candidates with transverse momenta $p_{T}$ greater than 35 $\mathrm{GeV}$ and $25 \mathrm{GeV}$, and at least one jet with $p_{T}>25$ $\mathrm{GeV}$ containing a $b$-hadron ( $b$-jet), identified using a $b$ tagging algorithm with an efficiency of $77 \%$ [10].

Selected events are sorted into two $t \bar{t} H$-enriched regions. The "Lep" region, targeting top quark decays in which at least one of the resulting $W$ bosons decays leptonically, requires events to have at least one isolated lepton (muon or electron) candidate with $p_{T}>15 \mathrm{GeV}$. The "Had" region targets hadronic top quark decays (as 
well as top quark decays to both hadronically decaying $\tau$-leptons and unreconstructed leptons) and requires events to have at least two additional jets with $p_{T}>25$ $\mathrm{GeV}$ and no selected lepton.

In each region, two boosted decision trees (BDTs) are trained: "Background Rejection BDT" and "CP BDT". The Background Rejection BDT is trained to separate $t \bar{t} H$-like events from background which are mainly nonresonant diphoton production processes $(\gamma \gamma+$ jets and $t \bar{t} \gamma \gamma)$. The $C P$ BDT is trained to separate $C P$-even from $C P$-odd couplings using $t \bar{t} H$ and $t H$ processes. The selected events are categorized using partitions of the twodimensional BDT space to enhance the analysis sensitivity. There are 20 categories in total: 12 in the Had region and 8 in the Lep region.

A simultaneous maximum-likelihood fit is performed to the diphoton invariant mass $\left(m_{\gamma \gamma}\right)$ spectra in all the categories with all the evaluated systematic uncertainties treated as nuisance parameters (NPs). Signal and background shapes are modeled by analytic functions. Figure 1 shows the distributions of the reconstructed masses for the diphoton system and primary top quark. The events are weighted by $\ln (1+S / B)$ with $S$ and $B$ being the fitted signal and background yields in the smallest $m_{\gamma \gamma}$ interval containing $90 \%$ of the signal in each category.

Assuming a $C P$-even coupling, the measured rate for $t \bar{t} H$ is $1.43_{-0.31}^{+0.33}$ (stat.) ${ }_{-0.15}^{+0.21}$ (sys.) times the SM expectation. The background-only hypothesis is rejected with an observed (expected) significance of $5.2 \sigma(4.4 \sigma)$. The $t H$ process is not observed and an upper limit of 12 times the SM expectation is set on its rate at $95 \%$ confidence level (CL).

The results of the fit for $\kappa_{t} \cos (\alpha)$ and $\kappa_{t} \sin (\alpha)$ are derived with $H \rightarrow \gamma \gamma$ branching ratio and the Higgs boson coupling to gluons constrained by the Run 2 Higgs boson coupling combination [11], and shown as contours in Figure 2. A limit on $\alpha$ is set without prior constraint on $\kappa_{t}$ in the fit: $|\alpha|>43^{\circ}$ is excluded at $95 \% \mathrm{CL}$. The expected exclusion is $|\alpha|>63^{\circ}$ under the $C P$-even hypothesis. A pure $C P$-odd coupling is excluded at $3.9 \sigma$.

\section{3. $H \rightarrow b \bar{b}$ in the $V H$ Production Mode}

$V H$ is the most sensitive production mode for detecting $H \rightarrow b \bar{b}$ decay. The leptonic decay of the vector boson enables efficient triggering and a significant reduction of the multi-jet background. Two analyses are performed: "Resolved Analysis" and "Boosted Analysis", which are depicted in the following two subsections.

\subsection{Resolved Analysis}

This analysis targets the event topologies containing exactly a pair of $b$-jets with radius parameter of $R=0.4$, referred to as small-radius (small- $R$ ) jets, to reconstruct the Higgs boson. Events are categorized into 0-, 1and 2-lepton channels (referred to as the $n$-lepton channels) depending on the number of selected electrons and muons, to target the $Z H \rightarrow v v b \bar{b}, W H \rightarrow \ell v b \bar{b}$ and $Z H \rightarrow \ell \ell b \bar{b}$ signatures, respectively. Events are further split into 2-jet or 3-jet regions, where the 3-jet category includes events with one or more untagged jets. In the 0 and 1-lepton channels, only one untagged jet is allowed, while in the 2-lepton channel any number of untagged jets are accepted in the 3-jet category. Since the signalto-background ratio increases for large $p_{T}^{V}$ (transverse momentum of the vector boson) values, the selected events are also sorted into two high- $p_{T}^{V}$ regions defined as $150 \mathrm{GeV}<p_{T}^{V}<250 \mathrm{GeV}$ and $p_{T}^{V}>250 \mathrm{GeV}$. In the 2-lepton channel, an additional medium- $p_{T}^{V}$ region with $75 \mathrm{GeV}<p_{T}^{V}<150 \mathrm{GeV}$ is included.

The three $n$-lepton channels, two jet categories and two (0-lepton, 1-lepton) or three (2-lepton) $p_{T}^{V}$ regions result in a total of 14 analysis regions. Each analysis region is further split into one signal region (SR) and two control regions (CRs) using a continuous selection on the angular differences between the two $b$-jets as a function of $p_{T}^{V}$, resulting in a total of 42 regions.

The BDT is used to improve the sensitivity of the analysis. It is trained to discriminate the $\mathrm{VH}$ signal from the background processes in eight regions, obtained by merging some of the 14 analysis regions. The BDT outputs, evaluated in each signal region, are used as final discriminating variables.

A binned likelihood fit is performed to the BDT outputs in all the categories. The effects of systematic uncertainties enter the likelihood as NPs. The normalizations of the dominant background $V+\mathrm{HF}$ (heavy flavor) and $t \bar{t}$ are left unconstrained in the likelihood, and determined by the fit. The $W H$ and $Z H$ production modes reject the background-only hypothesis with observed (expected) significances of $4.0 \sigma(4.1 \sigma)$ and $5.3 \sigma(5.1 \sigma)$, respectively. The total observed (expected) significance for $V H$ production mode is $6.7 \sigma(6.7 \sigma)$. Figure 3 shows the measured $V H$ cross-sections times the $H \rightarrow b \bar{b}$ and $V \rightarrow$ leptons branching fractions, $\sigma \times B$, together with the SM predictions in the reduced simplified template cross-section (STXS) [12] regions. The measurements are all consistent with the SM predictions with relative uncertainties varying from $30 \%$ in the highest $p_{T}^{V}$ region to $85 \%$ in the lowest $p_{T}^{V}$ region. The data statistical uncertainty is the largest single uncertainty, and the major systematic uncertainties arise from the background 

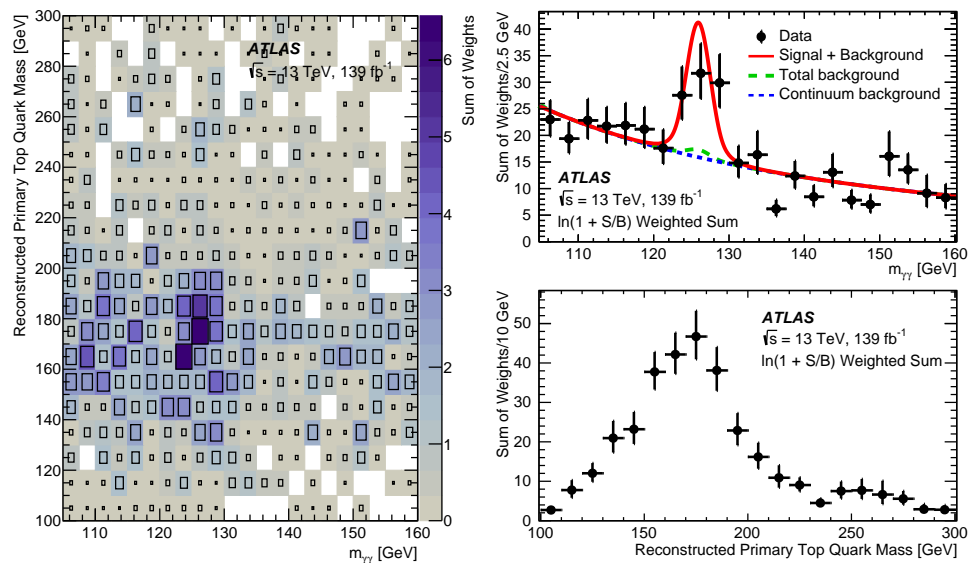

Figure 1: Distribution of reconstructed primary top quark mass versus reconstructed Higgs boson mass in the data events. The right panels show the projections onto the Higgs boson mass and primary top quark mass axes. In the upper panel, the fitted continuum background (blue), the total background including non- $t \bar{t} H / t H$ Higgs boson production (green), and the total fitted signal plus background (red) are shown [4].

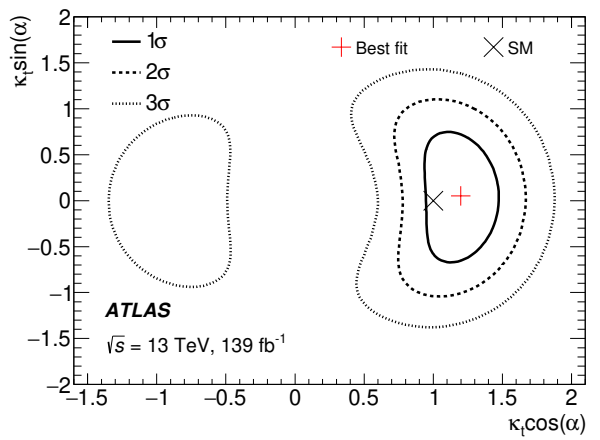

Figure 2: Two-dimensional likelihood contours for $\kappa_{t} \cos (\alpha)$ and $\kappa_{t} \sin (\alpha)$ with $g g F$ and $H \rightarrow \gamma \gamma$ constrained by the Higgs boson coupling combination [4].

modelling, $b$-tagging correction factors as well as jet energy scale calibration and resolution.

\subsection{Boosted Analysis}

This analysis aims for high $p_{T}$ Higgs boson regime, where the Higgs boson is reconstructed as a single large$R$ jet $(J)$ with $R=1.0$ and at least two track-jets associated. The large- $R$ jets are required to have $p_{T}>250$ $\mathrm{GeV}$, mass $m_{\mathrm{J}}>50 \mathrm{GeV}$ and pseudorapidity $|\eta|<2.0$. The leading two track-jets associated with $J$ are required to be $b$-tagged.

Events are categorized into 0-, 1- and 2-lepton channels depending on the number of selected electrons and muons. $10 \mathrm{SRs}$ and $4 \mathrm{CRs}$ are defined based on $p_{T}^{V}$ $\left(250<p_{T}^{V}<400 \mathrm{GeV}\right.$ or $\left.p_{T}^{V}>400 \mathrm{GeV}\right)$ and the number of small- $R$ jets not matched to $J$.

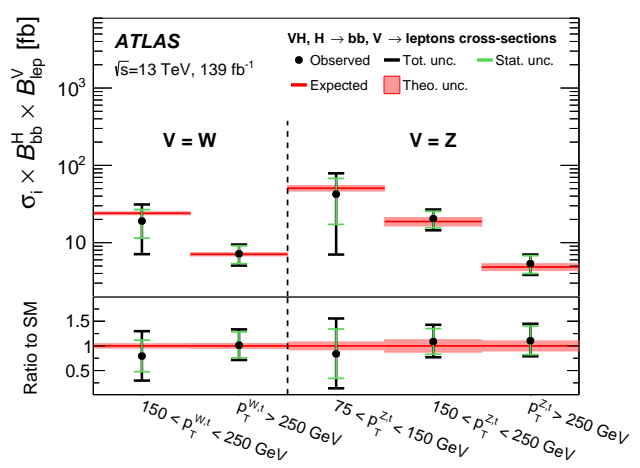

Figure 3: Measured $V H, V \rightarrow$ leptons cross-sections times the $H \rightarrow$ $b \bar{b}$ branching fraction in the reduced STXS scheme [5].

The results are obtained from a binned maximumprofile-likelihood fit to the observed $m_{\mathrm{J}}$ distributions in all the SRs and CRs. The normalizations of the dominant background $V+$ jets and $t \bar{t}$ are left unconstrained in the likelihood, and determined by the fit. The observed (expected) significance is $2.1 \sigma(2.7 \sigma)$ for $V H, H \rightarrow b \bar{b}$ process. Figure 4 shows the measured $V H$ cross-section times branching fractions $\sigma \times B$ in each STXS bin for the reduced scheme, together with the SM predictions. For these results, the largest uncertainty arises from data statistics. The major systematic uncertainty is related to the large-R jet calibration, particularly the $m_{\mathrm{J}}$ resolution.

\section{4. $H \rightarrow \mu^{+} \mu^{-}$}

The $H \rightarrow \mu^{+} \mu^{-}$decay offers the best opportunity to measure the Higgs boson interactions with a second- 


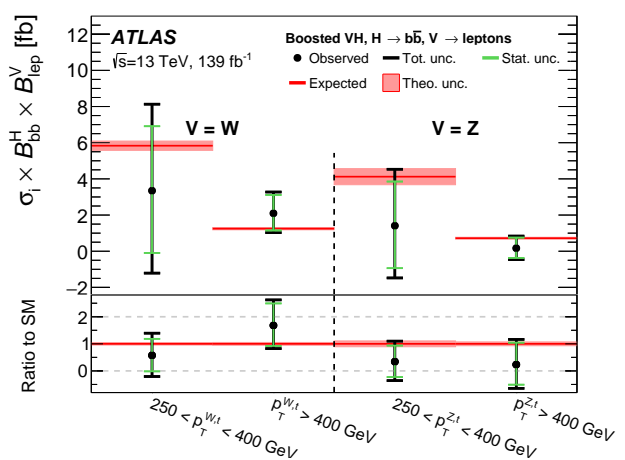

Figure 4: Measured $V H$ reduced stage-1.2 simplified template crosssections times the $H \rightarrow b \bar{b}$ and $V \rightarrow$ leptons branching fractions [6].

generation fermion at the LHC. This analysis selects events with two isolated muons with opposite charge and the leading muon is required to have $p_{T}>27 \mathrm{GeV}$. To increase the signal sensitivity, the selected events are classified into 20 mutually exclusive categories based on the event topology and BDT discriminants.

A category enriched in $t \bar{t} H$ events is defined in order to target the dileptonic or semileptonic decay of the $t \bar{t}$ system. Events are considered for this category if there is at least one lepton $(e$ or $\mu)$ with $p_{T}>15 \mathrm{GeV}$ in addition to the opposite-sign muon pair and at least one $b$-jet identified using a $b$-tagging algorithm with an efficiency of $85 \%$. A BDT is trained using simulated $t \bar{t} H, H \rightarrow \mu^{+} \mu^{-}$events as signal and simulated events from all SM background processes as background. A selection is applied to the BDT score to define one $t \bar{t} H$ enriched category.

Events not selected in the $t \bar{t} H$ category are considered for the $\mathrm{VH}$-enriched categories. The $\mathrm{VH}$ categories target signal events where the Higgs boson is produced in association with a leptonically decaying vector boson. Events are required to have at least one additional isolated lepton. Two BDTs are trained, separately for the three-lepton and four-lepton events, to discriminate between the simulated signal and background events. Based on the BDT scores, two categories are defined for the three-lepton events and one category is defined for the four-lepton events.

The events not selected in the $t \bar{t} H$ or $V H$ categories, are further classified into three channels according to the jet multiplicity: 0 -jet, 1 -jet and 2-jet, where the last includes events with two or more jets. In the 2-jet channel, a BDT is trained to disentangle signal events produced by VBF, used as signal sample in the training, from background events. Four VBF categories are defined based on this BDT classifier. The remaining events are

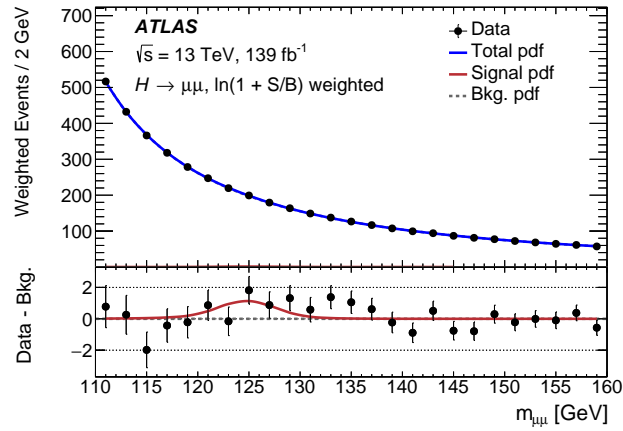

Figure 5: Dimuon invariant mass spectrum in all the analysis categories observed in data. Events and pdfs are weighted by $\ln (1+S / B)$, where $S$ are the observed signal yields and $B$ are the background yields derived from the fit to data in the $m_{\mu \mu}=120-130 \mathrm{GeV}$ window [7].

classified by three other BDTs (split by jet multiplicity), which are trained with both the $H \rightarrow \mu^{+} \mu^{-} \mathrm{ggF}$ and VBF production MC samples as signal. Four categories are defined in each of the three BDTs, resulting in twelve ggF categories in total.

The signal yield is obtained by a simultaneous binned maximum-likelihood fit to the dimuon invariant mass $m_{\mu \mu}$ distributions of the 20 categories in the range $110-$ $160 \mathrm{GeV}$. Analytic models are used in the fit to describe the $m_{\mu \mu}$ distributions for both the signal and background processes. Figure 5 shows the $m_{\mu \mu}$ spectra for all the analysis categories after the signal-plus-background fit. The best-fit value of the signal strength parameter, defined as the ratio of the observed signal yield to the one expected in the SM, is $\mu=1.2 \pm 0.6$, corresponding to an observed (expected) significance of $2.0 \sigma(1.7 \sigma)$ with respect to the hypothesis of no $H \rightarrow \mu^{+} \mu^{-}$signal. The best-fit values of the signal strength parameters for the five major groups of categories $(t \bar{t} H+V H$, ggF 0 -jet, 1 jet, 2-jet, and $\mathrm{VBF}$ ) together with the combined value are shown in Figure 6.

\section{5. $H \rightarrow e^{+} e^{-}$}

This is the first ATLAS search for $H \rightarrow e^{+} e^{-}$process, which has very small branching fraction (around $5 \times 10^{-9}$ ). Events are selected by requiring exactly two isolated electrons with opposite charge, and the leading electron needs to have $p_{T}>27 \mathrm{GeV}$.

To improve the overall sensitivity of this search, selected events are divided into seven categories. First, a category enriched in events from VBF production is defined by selecting those containing two jets with pseudorapidities of opposite signs, a pseudorapidity separa- 


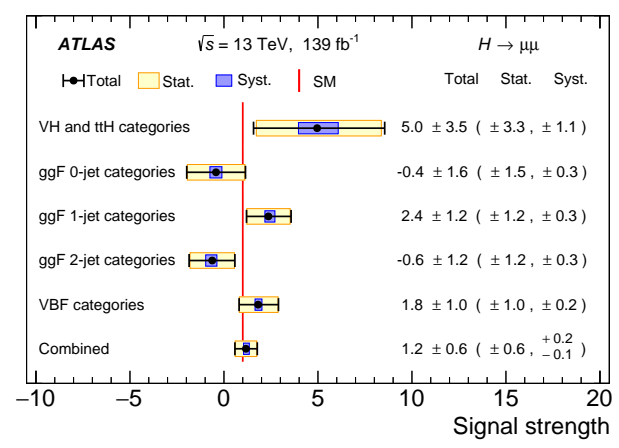

Figure 6: The best-fit values of the signal strength parameters for the five major groups of categories together with the combined value [7].

tion $\left|\Delta \eta_{j j}\right|>3$ and a dijet invariant mass $m_{j j}>500 \mathrm{GeV}$. Events that fail to meet the criteria of the VBF category are classified as "Central" if the pseudorapidities of both leptons are $\left|\eta^{e}\right|<1$ or as "Non-central" otherwise. For each of these two regions, three categories are defined based on the dielectron transverse momentum $p_{T}^{e e}$ : "Low $p_{T}^{e e}$ " $\left(p_{T}^{e e} \leq 15 \mathrm{GeV}\right)$, "Mid $p_{T}^{e e}$ " $\left(15<p_{T}^{e e} \leq 50\right.$ $\mathrm{GeV})$, and "High $p_{T}^{e e}$ " $\left(p_{T}^{e e}>50 \mathrm{GeV}\right)$.

The signal yield is obtained by a simultaneous binned maximum-likelihood fit to the dielectron invariant mass spectra of the seven categories in the range $110-$ $160 \mathrm{GeV}$. The data and expectation for all categories summed together are shown in Figure 7. No evidence of the $H \rightarrow e e$ process is found. The observed (expected) upper limit on the branching fraction is set as $3.6 \times 10^{-4}$ $\left(3.5 \times 10^{-4}\right)$ at the $95 \% \mathrm{CL}$.

\section{Summary}

The latest studies of the SM Higgs boson couplings to the fermions with $139 \mathrm{fb}^{-1}$ data are presented. All the measured results are consistent with the SM predictions, and they represent significant improvements on sensitivity and precision comparing with previous publications.

\section{References}

[1] ATLAS Collaboration, Observation of Higgs boson production in association with a top quark pair at the LHC with the ATLAS detector, Phys. Lett. B 716, 1 (2012).

[2] CMS Collaboration, Observation of a new boson at a mass of $125 \mathrm{GeV}$ with the CMS experiment at the LHC, Phys. Lett. B 716, 30 (2012).

[3] ATLAS Collaboration, The ATLAS experiment at the CERN Large Hadron Collider, JINST 3 (2008) S08003.

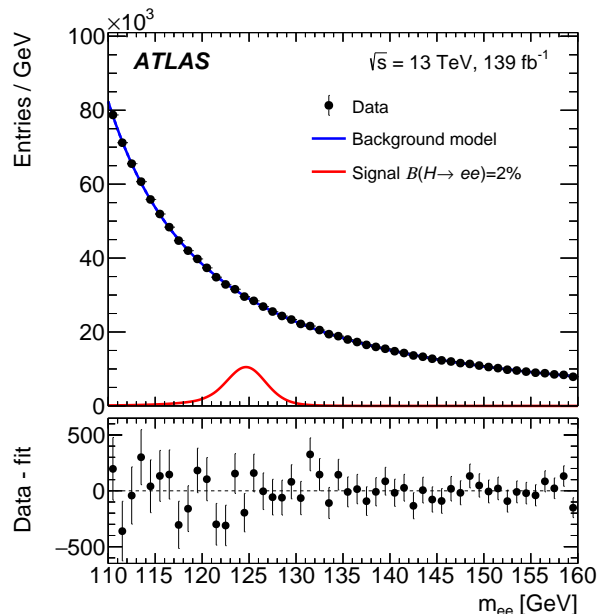

Figure 7: Dielectron invariant mass for all categories summed together compared with the background-only model. The signal parameterisations with branching fractions set to $\mathcal{B}\left(H \rightarrow e^{+} e^{-}\right)=2 \%$ are also shown (red line). The bottom panels show the difference between data and the background-only fit [8].

[4] ATLAS Collaboration, $C P$ Properties of Higgs Boson Interactions with Top Quarks in the $t \bar{t} H$ and $t H$ Processes Using $H \rightarrow \gamma \gamma$ with the ATLAS Detector, Phys. Rev. Lett. 125 (2020) 061802.

[5] ATLAS Collaboration, Measurements of $W H$ and $Z H$ production in the $H \rightarrow b \bar{b}$ decay channel in $p p$ collisions at $\sqrt{s}=13 \mathrm{TeV}$ with the ATLAS detector, arXiv:2007.02873.

[6] ATLAS Collaboration, Measurement of the associated production of a Higgs boson decaying into $b$-quarks with a vector boson at high transverse momentum in $p p$ collisions at $\sqrt{s}=13 \mathrm{TeV}$ with the ATLAS detector, arXiv:2008.02508.

[7] ATLAS Collaboration, A search for the dimuon decay of the Standard Model Higgs boson with the ATLAS detector, arXiv:2007.07830.

[8] ATLAS Collaboration, Search for the Higgs boson decays $H \rightarrow e^{+} e^{-}$and $H \rightarrow e \mu$ in $p p$ collisions at $\sqrt{s}=13 \mathrm{TeV}$ with the ATLAS detector, Phys. Lett. B 801 (2020) 135148.

[9] F. Demartin, F. Maltoni, K. Mawatari, B. Page, and M. Zaro, Higgs characterisation at NLO in QCD: $C P$ properties of the top-quark Yukawa interaction, Eur. Phys. J. C 74, 3065 (2014).

[10] ATLAS Collaboration, ATLAS $b$-jet identification performance and efficiency measurement with $t \bar{t}$ events in $p p$ collisions at $\sqrt{s}=13 \mathrm{TeV}$, Eur. Phys. J. C 79 (2019) 970.

[11] ATLAS Collaboration, Combined measurements of Higgs boson production and decay using up to $80 \mathrm{fb}^{-1}$ of proton-proton collision data at $\sqrt{s}=13 \mathrm{TeV}$ collected with the ATLAS experiment, Phys. Rev. D 101, 012002 (2020).

[12] ATLAS Collaboration, Measurement of $V H, H \rightarrow b \bar{b}$ production as a function of the vector-boson transverse momentum in $13 \mathrm{TeV} p p$ collisions with the ATLAS detector, JHEP 05 (2019) 141 